\title{
A correspondence of modular forms and applications to values of L-series
}

\author{
Nikolaos Diamantis", Michael Neururer and Fredrik Strömberg
}

*Correspondence:

nikolaos.diamantis@nottingham.ac.uk University of Nottingham,

Nottingham, UK

\begin{abstract}
A interpretation of the Rogers-Zudilin approach to the Boyd conjectures is established. This is based on a correspondence of modular forms which is of independent interest. We use the reinterpretation for two applications to values of $L$-series and values of their derivatives.
\end{abstract}

Keywords: L-functions, Derivatives of L-functions, Eisenstein series

\section{Introduction}

In [7], Zudilin outlines a method he developed with M. Rogers on which their proof of Boyd's conjectures is based. Here we reinterpret that method in terms of a correspondence of modular forms which is of independent interest. We use this reinterpretation to deduce some relations among values of L-functions and some relations involving values of the first derivative of an L-function.

The correspondence associates to a pair of functions $F_{1}, F_{2}$ and $s \in \mathbb{C}$ a new function $\Phi_{s}\left(F_{1}, F_{2}\right)$ which, when $F_{1}$ and $F_{2}$ are connected to modular forms, satisfies properties related to modularity for special values of $s$ (see Proposition 2.1). In the case where $F_{1}$ and $F_{2}$ are Eisenstein series, $\Phi_{s}\left(F_{1}, F_{2}\right)$ is again an Eisenstein series for certain $s$ and we make use of this fact in the two applications of our method described below.

Our main theorem, Theorem 3.2, connects the Mellin transform of the product $F_{1} F_{2}$ with the Mellin transform of functions associated to $F_{1}$ and $F_{2}$ via our correspondence. This is achieved using a simple "duality" relation (Lemma 3.1), which reformulates a key integration by substitution in Rogers-Zudilin's method. The content of the main theorem can be summarized as:

Theorem 1.1. Let $F_{1}$ and $F_{2}$ be functions on the upper half-plane given by

$$
F_{1}(z)=\sum_{m_{1}, n_{1} \geq 1} a_{1}\left(m_{1}\right) b_{1}\left(n_{1}\right) e^{2 \pi i m_{1} n_{1} z} \text { and } F_{2}(z)=\sum_{m_{2}, n_{2} \geq 1} a_{2}\left(m_{2}\right) b_{2}\left(n_{2}\right) e^{2 \pi i m_{2} n_{2} z}
$$

where we assume that the Fourier coefficients grow at most polynomially. For $j=1,2$ we set

$$
f_{j}(z)=\sum_{m_{j}, n_{j} \geq 1} b_{j}\left(n_{j}\right) e^{2 \pi i m_{j} n_{j} z} \text { and } g_{j}(z)=\sum_{m_{j}, n_{j} \geq 1} a_{j}\left(m_{j}\right) e^{2 \pi i m_{j} n_{j} z} .
$$

(C) 2015 Diamantis et al. Open Access This article is distributed under the terms of the Creative Commons Attribution 4.0 International License (http://creativecommons.org/licenses/by/4.0/), which permits unrestricted use, distribution, and reproduction in any medium, provided you give appropriate credit to the original author(s) and the source, provide a link to the Creative Commons license, and indicate if changes were made. 
Then we have the following relation between Mellin transforms

$$
\mathcal{M}\left(F_{1} \cdot F_{2} \mid W_{N}\right)(s)=\mathcal{M}\left(\Phi_{s+1}\left(f_{1}, f_{2}\right) \cdot\left(\Phi_{-s+1}\left(g_{2}, g_{1}\right) \mid W_{N}\right)\right)(s) \quad \text { for all } s \in \mathbb{C},
$$

where $W_{N}$ is the Fricke involution, | is the usual weight 0 "slash" operator and $\Phi_{s}(f, g)$ is a certain function associated to fand $g$ which will be described in Section 2.

We discuss two applications of this reinterpretation: Firstly we establish a relation among critical values of L-functions of products of Eisenstein series:

Theorem 1.2. Let $\chi_{1}, \chi_{2}$ and $\psi_{1}, \psi_{2}$ be pairs of non-trivial primitive Dirichlet characters modulo $M_{1}, M_{2}$ and $N_{1}, N_{2}$, respectively. Let $k \geq 1, l \geq 2$ such that $\left(\chi_{1} \cdot \chi_{2}\right)(-1)=(-1)^{l}$ and $\left(\psi_{1} \cdot \psi_{2}\right)(-1)=(-1)^{k}$. For a positive integer $r$ we set

$$
E_{k}^{\psi_{1}, \psi_{2}, r}(z)=2 \sum_{m, n \geq 1} \psi_{1}(m) \psi_{2}(n) n^{k-1} e^{2 \pi i n m r z}
$$

with an analogous definition for $E_{l}^{\chi_{1}, \chi_{2}, r}$. Then for an integer $j \in\{1, \ldots, k+l-1\}$ such that $\left(\chi_{1} \cdot \psi_{1}\right)(-1)=(-1)^{k-j}$ we have

$$
\Lambda\left(E_{l}^{\chi_{1}, \chi_{2}} \cdot E_{k}^{\bar{\psi}_{2}, \bar{\psi}_{1}, M_{1} M_{2}}, j\right)=C \cdot \Lambda\left(E_{j}^{\chi_{1}, \psi_{2}} \cdot E_{k+l-j}^{\bar{\chi}_{2}, \bar{\psi}_{1}, M_{1} N_{2}}, l\right)
$$

where $C$ is an explicit algebraic number given in section 4 and $\Lambda(E, s)$ denotes the completed L-function of $E$ as in (2).

This is extended to a more general class of characters in the second author's PhD thesis (in preparation). Since the space of modular forms of weight $k \geq 4$ is generated by products of such Eisenstein series when the level is prime [5], this result implies relations among critical values of L-functions for more general classes of modular forms than is at first apparent. A further extension of the main theorem of [5] to modular forms of squarefree level is implied by work in preparation by M. Dickson and the second author. Besides the independent interest of relations among critical values of different $L$-functions, possible applications we are currently exploring include vanishing and algebraicity results for values of L-functions, and the formulation of a general result of this nature specifically for cusp forms.

A second application exhibits a relation between derivatives of L-function of weight 2 modular forms at 1 and values of L-functions of weight 1 modular forms at 1 . The precise statement will be given in Theorem 5.2 but its content can be sketched in the following form.

Theorem 1.3. If E is in a certain subspace of the weight 2 Eisenstein space on $\Gamma_{1}(N)$, then

$$
\Lambda^{\prime}(E, 1)=\Lambda(\tilde{E}, 1)+C
$$

for an explicitly determined constant $C$ and an explicit element $\tilde{E}$ in the weight 1 Eisenstein space.

We stress that since L-functions of Eisenstein series can be expressed in terms of Dirichlet L-functions, such relations, once identified, can be verified directly. However, the proof gives a method to determine, for each given series $E$, a linear combination of weight 1 Eisenstein series whose L-function at 1 satisfies an explicit relation with $L^{\prime}(E, 1)$. This method is based on a combination of our reformulation of Rogers-Zudilin's method and Goldfeld's expression for derivatives of $L$-functions (cf. [3]). 
Furthermore the existence of an explicit, and fairly general relation between derivatives of L-functions and non-critical values of L-functions in the case of Eisenstein series, raises the question of whether analogous relations might exist in the case of cusp forms. In that case, such relations should not be possible to deduce by direct methods.

\section{A correspondence of modular forms}

We begin by fixing notation which will be used in the sequel. For a holomorphic function $g$ which is exponentially decaying at 0 and $\infty$ we denote the Mellin transform of $g$ by

$$
\mathcal{M} g(s):=\int_{0}^{\infty} g(i t) t^{s} \frac{d t}{t}
$$

If $g$ is any function defined on the upper half plane $\mathfrak{H}, k \in \mathbf{Z}$ and $\left(\begin{array}{ll}a & b \\ c & d\end{array}\right) \in \mathrm{SL}_{2}(\mathbb{R})$ then we define the weight $k$ slash operator by

$$
\left(\left.g\right|_{k}\left(\begin{array}{ll}
a & b \\
c & d
\end{array}\right)\right)(z)=(c z+d)^{-k} g\left(\frac{a z+b}{c z+d}\right) .
$$

When we omit the index $k$ we mean the weight 0 slash operator. The Fricke involution, $W_{N}=\left(\begin{array}{ll}0 & -\sqrt{N}^{-1} \\ \sqrt{N} & 0\end{array}\right)$, acts on functions on the upper half plane via the slash operator. It is well-known that $W_{N}$ preserves the space of modular forms of level $N$ and weight $k$ when it acts via the weight $k$ slash operator. We also have the following elementary but useful relationship between the weight $k$ and the weight 0 action on an arbitrary function on the upper half plane

$$
\mathcal{M}\left(\left.g\right|_{k} W_{N}\right)(s)=(\sqrt{N} i)^{-k} \mathcal{M}\left(g \mid W_{N}\right)(s-k) .
$$

Let $g(z)=\sum_{n=0}^{\infty} a(n) e^{2 \pi i n z}$ be a modular form for $\Gamma_{1}(N)$. For $\operatorname{Re}(s)$ large enough the $L$-series $L(g, s)=\sum_{n=1}^{\infty} a(n) n^{-s}$ converges absolutely and for such $s$ we define the completed $L$-function of $g$ by

$$
\Lambda(g, s)=\Gamma(s)\left(\frac{\sqrt{N}}{2 \pi}\right)^{s} L(g, s) .
$$

According to ([4], §7), $\Lambda(g, s)$ has meromorphic continuation to $s \in \mathbb{C}$ with possible simple poles at 0 and $k$, with residues $-a(0)$ and $i^{k} b(0)$ respectively, where $b(0)$ is the constant term of $\left.g\right|_{k} W_{N}$. If $g$ decays exponentially at 0 and $\infty$ we have the formula

$$
\Lambda(g, s)=N^{s / 2} \mathcal{M}(g)(s) .
$$

Let $f_{1}(z)=\sum_{m_{1}=0}^{\infty} \alpha\left(m_{1}\right) e^{2 \pi i m_{1} z}$ and $f_{2}(z)=\sum_{m_{2}=0}^{\infty} \beta\left(m_{2}\right) e^{2 \pi i m_{2} z}$ be functions on $\mathfrak{H}$. By applying the Möbius inversion formula we can rewrite $f_{1}$ and $f_{2}$ as double sums:

$$
f_{1}(z)=\sum_{m_{1}, n_{1} \geq 0}^{\infty} a\left(m_{1}\right) e^{2 \pi i m_{1} n_{1} z} \text { and } f_{2}(z)=\sum_{m_{2}, n_{2} \geq 0}^{\infty} b\left(m_{2}\right) e^{2 \pi i m_{2} n_{2} z}
$$

where $a(n):=\sum_{r \mid n} \alpha(r) \mu(n / r)$ and $b(n):=\sum_{r \mid n} \beta(r) \mu(n / r)$. Indeed, with this definition of $a(n)$, Möbius inversion formula implies that $\alpha(n)=\sum_{d \mid n} a(d)$ and thus

$$
f_{1}(z)=\sum_{m_{1}=0}^{\infty} \alpha\left(m_{1}\right) e^{2 \pi i m_{1} z}=\sum_{m_{1}=0}^{\infty} \sum_{d \mid m_{1}} a(d) e^{2 \pi i m_{1} z}=\sum_{d, n_{1} \geq 0}^{\infty} a(d) e^{2 \pi i d n_{1} z} .
$$


Likewise for $f_{2}(z)$.

We then define a new function $\Phi_{t}\left(f_{1}, f_{2}\right)$ on $\mathfrak{H}$ by the Fourier expansion

$$
\Phi_{t}\left(f_{1}, f_{2}\right)(z)=\sum_{m=1}^{\infty} \sum_{d \mid m} a(m / d) b(d) d^{t-1} e^{2 \pi m i z}=\sum_{m, n \geq 1} a(m) b(n) n^{t-1} e^{2 \pi i m n z} .
$$

This construction leads to a correspondence on spaces of Eisenstein series (see section 4 for definitions): Let $\psi$ and $\phi$ be non-trivial primitive Dirichlet characters $\bmod N_{1}$ and $N_{2}$, respectively. Assume that $\psi$ is odd and that the positive integer $l$ satisfies $\phi(-1)=(-1)^{l}$. If $f_{1}=E_{1}^{\psi, \mathbf{1}}, f_{2}=E_{l}^{\mathbf{1}, \phi}$ and $k$ is such that $k-l$ is even then the function $\Phi_{k}\left(f_{1}, f_{2}\right)=2 E_{k+l-1}^{\psi, \phi}$ is an Eisenstein series of weight $k+l-1$. This fact will be used in the proof of Theorem 1.2.

An analogous construction can be carried out when $f_{1}$ and $f_{2}$ are cusp forms of weight 1 , level $N$ and Dirichlet characters $v_{1}$ and $v_{2}$, respectively. Although we do not expect $\Phi_{t}\left(f_{1}, f_{2}\right)$ to be a modular form, Proposition 2.1 shows that if $t$ is even then all its twisted L-series satisfy the functional equations of a weight $t$ cusp form of level $N^{2}$ and character $v_{1} v_{2}$.

Specifically, for each prime $r \nmid N$, consider a primitive character $\psi$ of conductor $r$ such that $\psi(-1)=(-1)^{u}(u=0$ or 1$)$. For convenience $\psi$ can also stand for the trivial character $1 \bmod 1$. For $\operatorname{Re}(s) \gg 0$ consider

$$
\begin{aligned}
L\left(\Phi_{t}\left(f_{1}, f_{2}\right), \psi ; s\right) & =\sum_{m \geq 1} \frac{\psi(m)}{m^{s}}\left(\sum_{d \mid m} a\left(\frac{m}{d}\right) d^{t-1} b(d)\right) \\
& =\left(\sum_{m \geq 1} \frac{b(m) \psi(m)}{m^{s-t+1}}\right)\left(\sum_{l \geq 1} \frac{a(l) \psi(l)}{l^{s}}\right) .
\end{aligned}
$$

For the cusp forms $f_{1}$ and $f_{2}$ we define their L-series twisted by $\psi$ in the standard way, i.e.

$$
L\left(f_{1}, \psi ; s\right)=\sum_{n \geq 1} \frac{\psi(n) \alpha(n)}{n^{s}}=\sum_{n \geq 1} \frac{\psi(n)}{n^{s}}\left(\sum_{m \mid n} a(m)\right)=\left(\sum_{m \geq 1} \frac{a(m) \psi(m)}{m^{s}}\right) \cdot L(\psi, s)
$$

and similarly for $f_{2}$. From the definition of $L\left(\Phi_{t}\left(f_{1}, f_{2}\right), \psi ; s\right)$ we immediately deduce that

$$
L\left(\Phi_{t}\left(f_{1}, f_{2}\right), \psi ; s\right)=\frac{L\left(f_{1}, \psi ; s\right)}{L(\psi, s)} \frac{L\left(f_{2}, \psi ; s-t+1\right)}{L(\psi, s-t+1)} .
$$

Note that if $f_{1}$ and $f_{2}$ are Hecke eigenforms this equality implies that $L\left(\Phi_{t}\left(f_{1}, f_{2}\right), \psi ; s\right)$ has an Euler product representation. If we now define the completion of $L\left(\Phi_{t}\left(f_{1}, f_{2}\right), \psi ; s\right)$ as

$$
\Lambda\left(\Phi_{t}\left(f_{1}, f_{2}\right), \psi ; s\right):=\frac{\Gamma(s)(N r)^{s}}{(2 \pi)^{s}} L\left(\Phi_{t}\left(f_{1}, f_{2}\right), \psi ; s\right)
$$

then we have

Proposition 2.1. Let $f_{1}$ and $f_{2}$ be cusp forms of weight 1 , level $N$ and Dirichlet characters $v_{1}$ and $v_{2}$, respectively. Let $\psi$ be a primitive character of prime conductor $r \nmid N$. Then for even $t>1$ the completed L-series $\Lambda\left(\Phi_{t}\left(f_{1}, f_{2}\right), \psi ; s\right)$ satisfies the functional equation

$$
\Lambda\left(\Phi_{t}\left(f_{1}, f_{2}\right), \psi ; s\right)=(-1)^{t / 2} v_{1}(r) v_{2}(r) \psi\left(N^{2}\right) \tau(\psi) \Lambda\left(\Phi_{t}\left(\left.f_{2}\right|_{1} W_{N},\left.f_{1}\right|_{1} W_{N}\right), \bar{\psi} ; t-s\right),
$$

where 


$$
\tau(\psi):=\frac{1}{\sqrt{r}} \sum_{n} \psi(n) e^{2 \pi i \frac{n}{r}}
$$

is the Gauss sum of $\psi$.

Proof. We first express $\Lambda\left(\Phi_{t}\left(f_{1}, f_{2}\right), \psi ; s\right)$ in terms of the completed L-series,

$$
\Lambda(f, \psi ; s):=\frac{\Gamma(s)(r \sqrt{N})^{s}}{(2 \pi)^{s}} L(f, \psi ; s) \quad \text { and } \quad \Lambda(\psi, s):=\left(\frac{r}{\pi}\right)^{s / 2} \Gamma\left(\frac{s+u}{2}\right) L(\psi, s)
$$

where $u=0$ or 1 is determined by $\psi(-1)=(-1)^{u}$. We then have

$$
\begin{aligned}
& \Lambda\left(\Phi_{t}\left(f_{1}, f_{2}\right), \psi ; s\right)= \\
& \quad\left(\frac{N r}{\pi}\right)^{\frac{t-1}{2}} 2^{s-t+1} \frac{\Gamma((s-t+1+u) / 2) \Gamma((s+u) / 2)}{\Gamma(s-t+1)} \frac{\Lambda\left(f_{1}, \psi ; s\right)}{\Lambda(\psi ; s)} \frac{\Lambda\left(f_{2}, \psi ; s-t+1\right)}{\Lambda(\psi ; s-t+1)} .
\end{aligned}
$$

We recall the functional equations for the L-functions which appear in the expression above:

$$
\begin{aligned}
\Lambda\left(f_{j}, \psi ; s\right) & =i v_{j}(r) \psi(N) \tau(\psi)^{2} \Lambda\left(\left.f_{j}\right|_{1} W_{N}, \bar{\psi} ; 1-s\right), \quad(j=1,2) \quad \text { and } \\
\Lambda(\psi ; s) & =i^{-u} \tau(\psi) \Lambda(\bar{\psi} ; 1-s)
\end{aligned}
$$

(cf. [4], Thm. 7.5 and (12.7)). By using these functional equations we can rewrite the righthand side of (5) to obtain

$$
\Lambda\left(\Phi_{t}\left(f_{1}, f_{2}\right), \psi ; s\right)=\epsilon \cdot \Lambda\left(\Phi_{t}\left(\left.f_{2}\right|_{1} W_{N},\left.f_{1}\right|_{1} W_{N}\right), \bar{\psi} ; t-s\right)
$$

where

$\epsilon=2^{2 s-t} \frac{\Gamma((s-t+1+u) / 2) \Gamma((s+u) / 2) \Gamma(1-s)}{\Gamma((-s+1+u) / 2) \Gamma((-s+t+u) / 2) \Gamma(s-t+1)}(-1)^{u+1} v_{1}(r) v_{2}(r) \psi\left(N^{2}\right) \tau(\psi)^{2}$.

The final version of the functional equation now follows from the identity

$$
\frac{\Gamma((s-t+1+u) / 2) \Gamma((s+u) / 2) \Gamma(1-s)}{\Gamma((-s+1+u) / 2) \Gamma((-s+t+u) / 2) \Gamma(s-t+1)}=2^{t-2 s}(-1)^{t / 2+u+1},
$$

which is valid for even $t$ and can be shown using standard properties of the Gamma function, including the reflection and duplication formulas.

Remark. It follows immediately from (4) that $L\left(\Phi_{t}\left(f_{1}, f_{2}\right), s\right)$ has infinitely many poles (assuming the Grand Simplicity Hypothesis [6]) and therefore $\Phi_{t}\left(f_{1}, f_{2}\right)$ can not be a modular form. However, the extension of the converse theorem of [1] to general levels implies that $\Phi_{t}\left(f_{1}, f_{2}\right)$ is a modular integral.

\section{A reinterpretation of the method of Rogers-Zudilin}

The method of [7] relies crucially on a simple change of variables in an integral of the product of two series which leads to a product of two different functions. This part of the method can be expressed as the following simple "duality relation" involving the functions rather than their Fourier expansions. For a function $h$ on $\mathfrak{H}$ and $x \in \mathbb{Z}$, we set $h^{(x)}$ for the function defined by $h^{(x)}(z):=h(x z)$.

Lemma 3.1. Let $f, g: \mathfrak{H} \rightarrow \mathbb{C}$ be holomorphic functions with exponential decay at infinity and at most polynomial growth at 0 . For each $m, n \in \mathbb{N}$ and $s \in \mathbb{C}$ we have

$$
\mathcal{M}\left(f^{(m)} \cdot\left(g^{(n)} \mid W_{N}\right)\right)(s)=(n / m)^{s} \mathcal{M}\left(f^{(n)} \cdot\left(g^{(m)} \mid W_{N}\right)\right)(s) .
$$


Proof. From the growth conditions at infinity and 0 it follows that the product $f \cdot g \mid W_{N}$ has exponential decay at both infinity and 0 and thus the Mellin transforms on both sides are well-defined. By the change of variables $t \rightarrow(n / m) t$ we see that $\mathcal{M}\left(f^{(m)} \cdot g^{(n)} \mid W_{N}\right)(s)$ equals

$$
\int_{0}^{\infty} f(m i t) g\left(\frac{n i}{N t}\right) t^{s} \frac{d t}{t}=(n / m)^{s} \int_{0}^{\infty} f(n i t) g\left(\frac{m i}{N t}\right) t^{s} \frac{d t}{t}
$$

With the above lemma we obtain the following

Theorem 3.2. Let $F_{1}, F_{2}: \mathfrak{H} \rightarrow \mathbb{C}$ be given by the Fourier expansions

$$
F_{1}(z)=\sum_{m_{1}, n_{1} \geq 1} a_{1}\left(m_{1}\right) b_{1}\left(n_{1}\right) e^{2 \pi i m_{1} n_{1} z} \text { and } F_{2}(z)=\sum_{m_{2}, n_{2} \geq 1} a_{2}\left(m_{2}\right) b_{2}\left(n_{2}\right) e^{2 \pi i m_{2} n_{2} z} \text {, }
$$

where we assume, additionally, that the coefficients $a_{j}(n)$ and $b_{j}(n)$ grow at most polynomially in $n$. If we define the functions

$$
f_{j}(z)=\sum_{m_{j}, n_{j} \geq 1} b_{j}\left(n_{j}\right) e^{2 \pi i m_{j} n_{j} z} \text { and } g_{j}(z)=\sum_{m_{j}, n_{j} \geq 1} a_{j}\left(m_{j}\right) e^{2 \pi i m_{j} n_{j} z} \quad(j=1,2)
$$

then we have the following relation between Mellin transforms

$$
\mathcal{M}\left(F_{1} \cdot F_{2} \mid W_{N}\right)(s)=\mathcal{M}\left(\Phi_{s+1}\left(f_{1}, f_{2}\right) \cdot\left(\Phi_{-s+1}\left(g_{2}, g_{1}\right) \mid W_{N}\right)\right)(s) \quad \text { for all } s \in \mathbb{C} .
$$

Proof. Set $h_{j}(z):=\sum_{n_{j} \geq 1} b_{j}\left(n_{j}\right) e^{2 \pi i n_{j} z}$ for $j=1,2$. By comparing with $\int_{1}^{\infty} x^{k} e^{-y x} d x$, we see that for any positive integer $k$ we have that $\sum_{n=1}^{\infty} n^{k} e^{-n y}$ is bounded by a constant times $e^{-y}$ as $y \rightarrow \infty$ and by a constant times $y^{-(k+1)}$ as $y \rightarrow 0$. It follows then from the growth conditions on $b_{j}(n)$ that $h_{1}$ and $h_{2}$ both have exponential decay at infinity and at most polynomial growth at 0 . Hence Lemma 3.1 implies

$$
\begin{aligned}
\mathcal{M}\left(h_{1}^{\left(m_{1}\right)} \cdot h_{2}^{\left(m_{2}\right)} \mid W_{N}\right)(s) & =\left(\frac{m_{2}}{m_{1}}\right)^{s} \int_{0}^{\infty} h_{1}\left(m_{2} i t\right) \cdot h_{2}\left(\frac{i m_{1}}{N t}\right) t^{t} \frac{d t}{t} \\
& =\left(\frac{m_{2}}{m_{1}}\right)^{s} \int_{0}^{\infty} \sum_{n_{1}, n_{2} \geq 1} b_{1}\left(n_{1}\right) b_{2}\left(n_{2}\right) e^{-\frac{2 \pi m_{1} n_{2}}{N t}} e^{-2 \pi n_{1} m_{2} t} t^{s} \frac{d t}{t}
\end{aligned}
$$

The growth condition of $b_{j}$ justifies the interchange of integration and summation, so, upon the further change of variables $t \rightarrow\left(n_{2} / m_{2}\right) t$ we deduce that

$$
\begin{aligned}
\mathcal{M}\left(h_{1}^{\left(m_{1}\right)} \cdot h_{2}^{\left(m_{2}\right)} \mid W_{N}\right)(s) & =m_{1}^{-s} \int_{0}^{\infty} \sum_{n_{1}, n_{2} \geq 1} b_{1}\left(n_{1}\right) b_{2}\left(n_{2}\right) n_{2}^{s} e^{-\frac{2 \pi m_{1} m_{2}}{N t}} e^{-2 \pi n_{1} n_{2} t} t^{s} \frac{d t}{t} \\
& =m_{1}^{-s} \int_{0}^{\infty} \Phi_{s+1}\left(f_{1}, f_{2}\right)(i t) e^{\frac{-2 \pi m_{1} m_{2}}{N t}} t^{s} \frac{d t}{t} .
\end{aligned}
$$

The desired conclusion now follows from the fact that

$$
F_{1} \cdot F_{2} \mid W_{N}(z)=\sum_{m_{1}, m_{2} \geq 1} a_{1}\left(m_{1}\right) a_{2}\left(m_{2}\right) h_{1}^{\left(m_{1}\right)}(z) \cdot\left(h_{2}^{\left(m_{2}\right)} \mid W_{N}\right)(z) .
$$




\section{An application to products of Eisenstein series}

We recall the weight $k$ Eisenstein series $E_{k}^{\psi, \phi}$ assigned to primitive Dirichlet characters $\psi$ $\bmod N_{1}$ and $\phi \bmod N_{2}$ which satisfy $\psi \phi(-1)=(-1)^{k}$. Its Fourier expansion at infinity is given by

$$
E_{k}^{\psi, \phi}(z)=a_{k}^{\psi, \phi}+2 \sum_{m, n \geq 1} \psi(m) \phi(n) n^{k-1} e^{2 \pi i n m z},
$$

where the constant term is given by

$$
a_{k}^{\psi, \phi}=\left\{\begin{array}{l}
\delta(\psi) L(1-k, \phi) \text { if } k>1 \text { and } \\
\delta(\psi) L(0, \phi)+\delta(\phi) L(0, \psi) \text { if } k=1 .
\end{array}\right.
$$

For $t \in \mathbb{R}$ we also consider the function $E_{k}^{\psi, \phi, t}$ given by $E_{k}^{\psi, \phi, t}(z)=E_{k}^{\psi, \phi}(t z)$.

In Section 4.8 of [2] it is shown that, for $k=1$ and $t \in \mathbb{N}$, each $E_{k}^{\psi, \phi, t}$ is a weight 1 modular form for $\Gamma_{0}\left(t N_{1} N_{2}\right)$ and character $\psi \phi$. Furthermore, for a fixed $N \in \mathbb{N}$, these Eisenstein series give a basis of the Eisenstein subspace $\mathcal{E}_{1}\left(\Gamma_{1}(N)\right)$. To be precise, if $A$ is the set of $(\{\psi, \phi\}, t)$ such that $\psi$ and $\phi$ are primitive Dirichlet characters modulo $N_{1}$ and $N_{2}$ such that $(\psi \phi)(-1)=-1$ and $t$ is a positive integer such that $t N_{1} N_{2} \mid N$ then the set

$$
\left\{E_{1}^{\psi, \phi, t} ;(\{\psi, \phi\}, t) \in A\right\}
$$

is a basis of $\mathcal{E}_{1}\left(\Gamma_{1}(N)\right)$. For convenience we will often write $E_{1}^{\psi}$ for $E_{1}^{\psi, \mathbf{1}, 1}$ and $a_{\psi}$ for $a_{1}^{\psi, \mathbf{1}}$ where 1 is the trivial character modulo 1 and we also consider the subspace

$$
\mathcal{E}_{1}^{\prime}\left(\Gamma_{1}(N)\right)=\left\langle\left\{E_{1}^{\psi, \mathbf{1}, 1} ;(\psi, \mathbf{1}, 1) \in A\right\}\right\rangle .
$$

The analogous statement for $k=2$ (Th. 4.6.2 of [2]) involves the set $B$ of $(\psi, \phi, t)$ such that $\psi$ and $\phi$ are primitive Dirichlet characters modulo $N_{1}$ and $N_{2}$ with $(\psi \phi)(-1)=1$, and $t$ is a positive integer such that $1<t N_{1} N_{2} \mid N$. In this case the set

$$
\left\{E_{2}^{\psi, \phi, t} ;(\psi, \phi, t) \in B\right\} \cup\left\{E_{2}^{\mathbf{1}, \mathbf{1}, 1}-t E_{2}^{\mathbf{1}, \mathbf{1}, t} ; t \mid N\right\}
$$

forms a basis of $\mathcal{E}_{2}\left(\Gamma_{1}(N)\right)$. We also consider the smaller subspace

$$
\mathcal{E}_{2}^{\prime}\left(\Gamma_{1}(N)\right)=\left\langle\left\{E_{2}^{\psi, \phi, 1} ;(\psi, \phi, 1) \in B ; \phi, \psi \text { odd }\right\}\right\rangle .
$$

In the sequel we will often use the following identity

$$
\left.E_{k}^{\psi, \phi}\right|_{k} W_{t N_{1} N_{2}}=(-1)^{k} \tau(\psi) \tau(\phi)\left(\frac{N_{2}}{N_{1}}\right)^{\frac{k-1}{2}} t^{k / 2} E_{k}^{\bar{\phi}, \bar{\psi}, t},
$$

which is valid for any $t>0$ (see e.g. Th. 12.1 of [4] for a version with $t=1$ ).

We can now use Theorem 3.2 to prove Theorem 1.2. Recall that $\psi_{i}$ and $\chi_{i}(i=1,2)$ are non-trivial primitive characters modulo $N_{i}$ and $M_{i}$ such that $\left(\chi_{1} \cdot \chi_{2}\right)(-1)=(-1)^{l}$ and $\left(\psi_{1} \cdot \psi_{2}\right)(-1)=(-1)^{k}$. We will regard both Eisenstein series $E_{l}^{\chi_{1}, \chi_{2}}$ and $E_{k}^{\psi_{1}, \psi_{2}}$ as modular forms of level $M N$ where $M=M_{1} M_{2}$ and $N=N_{1} N_{2}$. It follows immediately from (7) that

$$
(-1)^{k} \tau\left(\psi_{1}\right) \tau\left(\psi_{2}\right)\left(\frac{N_{2}}{N_{1}}\right)^{\frac{k-1}{2}} M^{k / 2} \Lambda\left(E_{l}^{\chi_{1}, \chi_{2}} \cdot E_{k}^{\bar{\psi}_{2}, \bar{\psi}_{1}, M}, j\right)=\Lambda\left(E_{l}^{\chi_{1}, \chi_{2}} \cdot\left(\left.E_{k}^{\psi_{1}, \psi_{2}}\right|_{k} W_{M N}\right), j\right) .
$$

Using the definition of the completed L-function and (1), we see that the right-hand side is

$$
(\sqrt{M N})^{j-k_{i}-k} \mathcal{M}\left(E_{l}^{\chi_{1}, \chi_{2}} \cdot\left(E_{k}^{\psi_{1}, \psi_{2}} \mid W_{M N}\right)\right)(j-k) .
$$


We are now in a position to apply Theorem 3.2 with Fourier expansions given by $a_{1}\left(m_{1}\right)=\chi_{2}\left(m_{1}\right) m_{1}^{l-1}, b_{1}\left(n_{1}\right)=\chi_{1}\left(n_{1}\right), a_{2}\left(m_{2}\right)=\psi_{1}\left(m_{2}\right), b_{2}\left(n_{2}\right)=\psi_{2}\left(n_{2}\right) n_{2}^{k-1}, s=j-k$, and we conclude that $\mathcal{M}\left(E_{l}^{\chi_{1}, \chi_{2}} \cdot\left(E_{k}^{\psi_{1}, \psi_{2}} \mid W_{M N}\right)\right)(j-k)$ can be expressed as

$$
\begin{array}{r}
4 \mathcal{M}\left(\Phi_{j-k+1}\left(f_{1}, f_{2}\right) \cdot\left(\Phi_{k-j+1}\left(g_{2}, g_{1}\right) \mid W_{M N}\right)\right)(j-k)=\mathcal{M}\left(E_{j}^{\chi_{1}, \psi_{2}} \cdot\left(E_{k+l-j}^{\psi_{1}, \chi_{2}} \mid W_{M N}\right)\right)(j-k) \\
=(\sqrt{M N})^{k-j} i^{k+l-j} \Lambda\left(E_{j}^{\chi_{1}, \psi_{2}} \cdot\left(\left.E_{k+l-j}^{\psi_{1}, \chi_{2}}\right|_{k+l-j} W_{M N}\right)\right)(l) .
\end{array}
$$

Finally, after another application of (7), we obtain the conclusion of Theorem 1.2:

$$
\Lambda\left(E_{l}^{\chi_{1}, \chi_{2}} \cdot E_{k}^{\bar{\psi}_{2}, \bar{\psi}_{1}, M}, j\right)=C \cdot \Lambda\left(E_{j}^{\chi_{1}, \psi_{2}} \cdot E_{k+l-j}^{\bar{\chi}_{2}, \bar{\psi}_{1}, M_{1} N_{2}}, l\right)
$$

where

$$
C=(-i)^{l-j} \tau\left(\chi_{2}\right) \tau\left(\psi_{2}\right)^{-1} M_{1}^{\frac{l-j}{2}} M_{2}^{\frac{l-j-1}{2}} N_{1}^{-\frac{l-j}{2}} N_{2}^{\frac{l-j+1}{2}} .
$$

\section{Application to derivatives of $\boldsymbol{L}$-functions}

Let $\psi$ and $\phi$ be odd, primitive Dirichlet characters modulo $N_{1}$ and $N_{2}$ respectively. Using the notation of the last section we set

$$
E_{1}^{\psi}:=E_{1}^{\psi, \mathbf{1}}, a_{\psi}:=a_{1}^{\psi, \mathbf{1}}, \text { and } f_{r}^{\psi, \phi}:=\frac{\sqrt{N}}{4}\left(E_{1}^{\psi}-a_{\psi}\right) \cdot\left(\left.\left(E_{1}^{\phi, r}-a_{\phi}\right)\right|_{1} W_{N}\right), N=N_{1} N_{2} \text {. }
$$

The goal of this section is to evaluate a particular linear combination of the special values $\mathcal{M}\left(f_{r}^{\psi, \phi}\right)(2)$ in two different ways thereby obtaining a relation between values and derivatives of certain L-functions. We first observe that for a fixed positive integer $r$ we can write

$$
\mathcal{M}\left(f_{r}^{\psi, \phi}\right)(2)=\frac{1}{4 i} \mathcal{M}\left(\left(E_{1}^{\psi}-a_{\psi}\right) \cdot\left(\left(E_{1}^{\phi, r}-a_{\phi}\right) \mid W_{N}\right)\right)(1) .
$$

Since we now have a weight 0 action in the right-hand side we can use Theorem 3.2 with

$$
s=1, a_{1}(n)=1, b_{1}(n)=\psi(n), a_{2}(n)=\delta_{r}(n), b_{2}(n)=\phi(n)
$$

where $\delta_{r}(n)=1$ if $r \mid n$ and 0 otherwise. This implies that $\mathcal{M}\left(f_{r}^{\psi, \phi}\right)$ (2) equals

$$
\frac{1}{i} \mathcal{M}\left(\Phi_{2}\left(f_{1}, f_{2}\right) \cdot \Phi_{0}\left(g_{2}, g_{1}\right) \mid W_{N}\right)(1)=\frac{1}{2 i} \mathcal{M}\left(E_{2}^{\psi, \phi}(i t) \cdot \sum_{n_{1}, n_{2} \geq 1} \frac{1}{n_{1}} e^{\frac{-2 \pi r n_{1} n_{2}}{N t}}\right) \text { (1). }
$$

From the following well-known expression for the logarithm of the Dedekind eta function

$$
\sum_{m, n \geq 1} \frac{1}{n} e^{\frac{-2 \pi r m n}{N u}}=-\sum_{m \geq 1} \log \left(1-e^{\frac{-2 \pi r m}{N u}}\right)=-\log \left(\eta(r i /(N u)) e^{\frac{2 \pi r}{24 \cdot N u}}\right),
$$

we deduce that

$$
\mathcal{M}\left(f_{r}^{\psi, \phi}\right)(2)=\frac{i}{2} \int_{0}^{\infty} E_{2}^{\psi, \phi}(i u) \log \left(\eta(r i /(N u)) e^{\frac{2 \pi r}{24 \cdot N u}}\right) d u .
$$


The integral above is well-defined since $E_{2}^{\psi, \phi}$ decays exponentially at both $\infty$ and 0 . The decay at infinity is immediate since $\psi$ is not trivial and the decay at 0 follows from (7). By using (7) to rewrite $f_{r}^{\bar{\phi}, \bar{\psi}}$ it follows from (9) that

$$
\begin{aligned}
\mathcal{M}\left(F_{r}^{\psi, \phi}\right)(2) & =\frac{i}{2} \int_{0}^{\infty}\left(\left.E_{2}^{\psi, \phi}\right|_{2}\left(1+W_{N}\right)\right)(i u) \log \left(\eta(r i /(N u)) e^{\frac{2 \pi r}{24 \cdot N u}}\right) d u \\
& \left.=-\frac{i}{2} \int_{0}^{\infty}\left(\left.E_{2}^{\psi, \phi}\right|_{2}\left(1+W_{N}\right)\right)(i u) \log (\eta(\text { riu })) e^{\frac{2 \pi r u}{24}}\right) d u
\end{aligned}
$$

where

$$
F_{r}^{\psi, \phi}:=f_{r}^{\psi, \phi}+\sqrt{\frac{N_{2}}{N_{1}}} \tau(\psi) \tau(\phi) f_{r}^{\bar{\phi}, \bar{\psi}} .
$$

It is clear from (10) that we can find a linear combination of $F_{r}^{\psi, \phi}$, s such that the exponentials inside the logarithm on the right-hand side are eliminated:

$$
\begin{aligned}
\mathcal{M}\left(\left(N_{1}+N_{2}\right)\left(F_{1}^{\psi, \phi}+F_{N}^{\psi, \phi}\right)\right. & \left.-(1+N)\left(F_{N_{1}}^{\psi, \phi}+F_{N_{2}}^{\psi, \phi}\right)\right)(2)= \\
& -\frac{i}{2} \int_{0}^{\infty}\left(\left.E_{2}^{\psi, \phi}\right|_{2}\left(1+W_{N}\right)\right)(i u) \log (V(i u)) d u,
\end{aligned}
$$

where

$$
V(z):=\frac{(\eta(z) \eta(N z))^{N_{1}+N_{2}}}{\left(\eta\left(N_{1} z\right) \eta\left(N_{2} z\right)\right)^{1+N}} .
$$

We will now proceed to evaluate the two sides of (11) separately.

\subsection{The right-hand side of (11)}

We first recall the principle behind Goldfeld's expression for derivatives of $L$-functions (cf. [3]):

Proposition 5.1. Let $f$ and $g$ be holomorphic functions on $\mathfrak{H}$ such that for some $N \in \mathbb{N}$ :

(i) $\left.f\right|_{2} W_{N}=f$

(ii) $\left.g\right|_{k} W_{N}= \pm g$, for some non-zero constant $k \in \mathbb{R}$. Then

$$
\int_{0}^{\infty} f(z) d z=0 \text { and } 2 \int_{0}^{\infty} f(i y) \log (g(i y)) d y=k \int_{0}^{\infty} f(i y) \log (y) d y .
$$

Proof. Condition (i) is equivalent to $f\left(W_{N} z\right) d\left(W_{N} z\right)=f(z) d z$. Therefore $\int_{0}^{\infty} f(z) d z=$ $\int_{W_{N} \infty}^{W_{N} 0} f(z) d z=\int_{\infty}^{0} f(z) d z$ and hence $\int_{0}^{\infty} f(z) d z=0$. Similarly, we see that

$$
\begin{aligned}
\int_{0}^{\infty} f(z) \log (g(z)) d z & =\int_{W_{N} \infty}^{W_{N} 0} f(z) \log (g(z)) d z=\int_{\infty}^{0} f(z) \log \left(g\left(W_{N} z\right)\right) d z \\
& =\int_{\infty}^{0} f(z) \log (g(z)) d z+i k \int_{\infty}^{0} f(i y) \log (y) d y+c^{\prime} \int_{0}^{\infty} f(z) d z
\end{aligned}
$$

for some $c^{\prime} \in \mathbb{C}$. This equality, together with $\int_{0}^{\infty} f(z) d z=0$, implies the conclusion.

Since Proposition 5.1 holds for $f=\left.E_{2}^{\psi, \phi}\right|_{2}\left(1+W_{N}\right)$ and $g=V$ with $k=N_{1}+N_{2}-1-N$, we deduce that

$$
\int_{0}^{\infty} f(i u) \log (V(i u)) d u=\frac{k}{2} \int_{0}^{\infty} f(i u) \log (u) d u=\frac{k}{2}(\mathcal{M} f)^{\prime}(1) .
$$


By using Proposition 5.1 together with (3) we can express the the right-hand side of (12) as

$$
\frac{\left(N_{1}-1\right)\left(1-N_{2}\right)}{2 \sqrt{N}} \Lambda^{\prime}\left(\left.E_{2}^{\psi, \phi}\right|_{2}\left(1+W_{N}\right), 1\right) .
$$

If $h$ is a modular form of weight 2 and level $N$ it is easy to see from the functional equation of $\Lambda(h, s)$ that $\Lambda^{\prime}\left(\left.h\right|_{2}\left(1+W_{N}\right), 1\right)=2 \Lambda^{\prime}(h, 1)$. It follows that the right-hand side of (11) equals

$$
\frac{i\left(N_{1}-1\right)\left(N_{2}-1\right)}{2 \sqrt{N}} \Lambda^{\prime}\left(E_{2}^{\psi, \phi}, 1\right) .
$$

\subsection{The left-hand side of (11)}

To compute the left-hand side of (11) we first express $\mathcal{M}\left(f_{r}^{\psi, \phi}\right)(s)$ in terms of completed L-functions by applying the Riemann trick:

$$
\mathcal{M}\left(f_{r}^{\psi, \phi}\right)(s)=\int_{1 / \sqrt{N}}^{\infty} f_{r}^{\psi, \phi}(i t) t^{s} \frac{d t}{t}+N^{-s} \int_{1 / \sqrt{N}}^{\infty} f_{r}^{\psi, \phi}\left(\frac{i}{N t}\right) t^{-s} \frac{d t}{t} .
$$

We can rewrite $f_{r}^{\psi, \phi}(i t)$ as a linear combination of functions that decay exponentially at $\infty$ as follows

$$
\begin{aligned}
f_{r}^{\psi, \phi}(i t)=\frac{\sqrt{N}}{4}\left(\left(E_{1}^{\psi}(i t)-a_{\psi}\right) \frac{a_{\phi} i}{\sqrt{N} t}\right. & -\left(\left(\left.\left(\left(E_{1}^{\psi} \mid{ }_{1} W_{N}\right) \cdot E_{1}^{\phi, r}\right)\right|_{2} W_{N}\right)(i t)+a_{\psi} b_{\phi}\right) \\
& \left.-a_{\psi}\left(\left(\left.E_{1}^{\phi, r}\right|_{1} W_{N}\right)(i t)-b_{\phi}\right)\right),
\end{aligned}
$$

where $b_{\phi}$ is the constant term of the Fourier expansion of $E_{1}^{\phi, r} \mid W_{N}$. Here we have used the fact that $E_{1}^{\psi} \cdot\left(\left.E_{1}^{\phi, r}\right|_{1} W_{N}\right)=-\left.\left(\left(\left.E_{1}^{\psi}\right|_{1} W_{N}\right) \cdot E_{1}^{\phi, r}\right)\right|_{2} W_{N}$ and we have a similar expression for $\left.f_{r}^{\psi, \phi}\right|_{2} W_{N}$. By substituting (15) and the analogue for $\left.f_{r}^{\psi, \phi}\right|_{2} W_{N}$ into (14) and using the integral representation of the completed L-series of a, not necessarily cuspidal, modular form (e.g. Th. 7.3. of [4]), we deduce that

$$
\begin{aligned}
\mathcal{M}\left(f_{r}^{\psi, \phi}\right)(s) & =\frac{N^{(1-s) / 2}}{4}\left(\Lambda\left(\left(\left.E_{1}^{\psi}\right|_{1} W_{N}\right) \cdot E_{1}^{\phi, r}, 2-s\right)+a_{\phi} i \Lambda\left(E_{1}^{\psi}, s-1\right)+a_{\psi} i \Lambda\left(E_{1}^{\phi, r}, 1-s\right)\right) \\
& =\frac{N^{(1-s) / 2}}{4}\left(-\tau(\psi) \sqrt{N_{2}} \Lambda\left(E_{1}^{\bar{\psi}, N_{2}} E_{1}^{\phi, r}, 2-s\right)+a_{\phi} i \Lambda\left(E_{1}^{\psi}, s-1\right)+a_{\psi} i \Lambda\left(E_{1}^{\phi, r}, 1-s\right)\right) .
\end{aligned}
$$

For the last equality we again used (7) and we have an analogous expression for $\mathcal{M}\left(f_{r}^{\bar{\phi}, \bar{\psi}}\right)(s)$.

We will now compute the value of the linear combination

$$
\mathcal{M}\left(\left(N_{1}+N_{2}\right)\left(F_{1}^{\psi, \phi}+F_{N}^{\psi, \phi}\right)-(1+N)\left(F_{N_{1}}^{\psi, \phi}+F_{N_{2}}^{\psi, \phi}\right)\right)(s)
$$

at $s=2$ by considering each of the three summands of (16) and the analogue for $f_{r}^{\bar{\phi}}, \bar{\psi}$.

First we treat the contributions from $L$-functions associated to products of Eisenstein series. In $\mathcal{M}\left(F_{1}^{\psi, \phi}+F_{N}^{\psi, \phi}\right)(s)$ they are

$$
\frac{N^{(1-s) / 2}}{4} \tau(\psi) \sqrt{N_{2}}\left[\Lambda\left(-E_{1}^{\bar{\psi}, N_{2}} E_{1}^{\phi, 1}+E_{1}^{\phi, N_{1}} E_{1}^{\bar{\psi}, 1}-E_{1}^{\bar{\psi}, N_{2}} E_{1}^{\phi, N}+E_{1}^{\phi, N_{1}} E_{1}^{\bar{\psi}, N}, 2-s\right)\right] .
$$

By using the trivial fact

$$
\Lambda(f, s)=a^{s} \Lambda\left(f^{(a)}, s\right),
$$


combined with $\left(E_{1}^{\phi, N_{1}} E_{1}^{\bar{\psi}, 1}\right)^{N_{2}}=E_{1}^{\phi, N} E_{1}^{\bar{\psi}, N_{2}}$ and $\left(E_{1}^{\phi, 1} E_{1}^{\bar{\psi}, N_{2}}\right)^{N_{1}}=E_{1}^{\phi, N_{1}} E_{1}^{\bar{\psi}, N}$, we obtain $\frac{N^{(1-s) / 2}}{4} \tau(\psi) \sqrt{N_{2}}\left[\Lambda\left(E_{1}^{\bar{\psi}, N_{2}} E_{1}^{\phi, 1}, 2-s\right)\left(N_{1}^{s-2}-1\right)+\Lambda\left(E_{1}^{\phi, N_{1}} E_{1}^{\bar{\psi}, 1}, 2-s\right)\left(1-N_{2}^{s-2}\right)\right]$.

Both $\Lambda\left(E_{1}^{\bar{\psi}, N_{2}} E_{1}^{\phi, 1}, 2-s\right)$ and $\Lambda\left(E_{1}^{\phi, N_{1}} E_{1}^{\bar{\psi}, 1}, 2-s\right)$ have a simple pole at $s=2$ with residue $-a_{\bar{\psi}} a_{\phi}$. Therefore (19) is equal to $\tau(\psi) a_{\bar{\psi}} a_{\phi} \log \left(N_{1} / N_{2}\right) / 4 \sqrt{N_{1}}$ at $s=2$. It is easy to verify that the contribution of products of Eisenstein series in $\mathcal{M}\left(F_{N_{1}}^{\psi, \phi}+F_{N_{2}}^{\psi, \phi}\right)$ (2) is exactly the same as that in $\mathcal{M}\left(F_{1}^{\psi, \phi}+F_{N}^{\psi, \phi}\right)(2)$ and hence the products of Eisenstein series contribute

$$
\frac{\tau(\psi) a_{\bar{\psi}} a_{\phi}\left(N_{1}+N_{2}-1-N\right)}{4 \sqrt{N_{1}}} \log \left(\frac{N_{1}}{N_{2}}\right)
$$

to $\mathcal{M}\left(\left(N_{1}+N_{2}\right)\left(F_{1}^{\psi, \phi}+F_{N}^{\psi, \phi}\right)-(1+N)\left(F_{N_{1}}^{\psi, \phi}+F_{N_{2}}^{\psi, \phi}\right)\right)(s)$.

Secondly, to compute the contribution of the terms coming from $E_{1}^{\phi, r}$ and $E_{1}^{\bar{\psi}, r}$ we apply (18) to $\Lambda\left(E_{1}^{\phi, r}, 1-s\right)$ and $\Lambda\left(E_{1}^{\bar{\psi}, r}, 1-s\right)$. Thus their contribution to $\mathcal{M}\left(F_{r}^{\phi, \psi}\right)(s)$ is

$$
\frac{N^{(1-s) / 2}}{4} r^{s-1}\left(a_{\psi} i \Lambda\left(E_{1}^{\phi}, 1-s\right)+a_{\bar{\phi}} i \sqrt{\frac{N_{2}}{N_{1}}} \tau(\psi) \tau(\phi) \Lambda\left(E_{1}^{\bar{\psi}}, 1-s\right)\right),
$$

which implies that the contribution of these terms to (17) at $s=2$ is 0 . We are now left with

$$
\begin{aligned}
& \mathcal{M}\left(\left(N_{1}+N_{2}\right)\left(F_{1}^{\psi, \phi}+F_{N}^{\psi, \phi}\right)-(1+N)\left(F_{N_{1}}^{\psi, \phi}+F_{N_{2}}^{\psi, \phi}\right)\right)(2)= \\
& \frac{\left(N_{1}+N_{2}-1-N\right)}{4 \sqrt{N_{1}}}\left[\tau(\psi) a_{\bar{\psi}} a_{\phi} \log \left(\frac{N_{1}}{N_{2}}\right)+\frac{i}{\sqrt{N_{2}}} \Lambda\left(\mathcal{E}^{\psi, \phi}, 1\right)\right]
\end{aligned}
$$

where $\mathcal{E}^{\psi, \phi}$ is given by $\mathcal{E}^{\psi, \phi}:=L(\phi, 0) E_{1}^{\psi}+\sqrt{\frac{N_{2}}{N_{1}}} \tau(\psi) \tau(\phi) L(\bar{\psi}, 0) E_{1}^{\bar{\phi}}$. We note that the last term of (20) is well-defined because the residues of $\Lambda\left(E_{1}^{\bar{\phi}}, s\right)$ and $\Lambda\left(E_{1}^{\psi}, s\right)$ at 1 cancel when we take the linear combination giving $\mathcal{E}^{\psi, \phi}$. Equations (13) and (20) together give

$$
i \sqrt{N_{2}} \tau(\psi) a_{\bar{\psi}} a_{\phi} \log \left(\frac{N_{1}}{N_{2}}\right)-\Lambda\left(\mathcal{E}^{\psi, \phi}, 1\right)=2 \Lambda^{\prime}\left(E_{2}^{\psi, \phi}, 1\right) .
$$

In the following theorem we extend the identity (21) to the spaces spanned by the Eisenstein series that occur in it. We denote by $j$ the map from $\mathcal{E}_{2}^{\prime}\left(\Gamma_{1}(N)\right)$ to $\mathcal{E}_{1}^{\prime}\left(\Gamma_{1}(N)\right) \otimes$ $\mathcal{E}_{1}^{\prime}\left(\Gamma_{1}(N)\right)$ induced by $E_{2}^{\psi, \phi} \mapsto\left(E_{1}^{\psi}, E_{1}^{\phi}\right)$.

Theorem 5.2. Let $\alpha: \mathcal{E}_{2}^{\prime}\left(\Gamma_{1}(N)\right) \rightarrow \mathbb{C}$ and $\beta: j\left(\mathcal{E}_{2}^{\prime}\left(\Gamma_{1}(N)\right)\right) \rightarrow \mathbb{C}$ be given by

$$
\begin{aligned}
\alpha(E) & =2 \Lambda^{\prime}(E, 1) \quad \text { and } \\
\beta\left(E_{1}^{\psi}, E_{1}^{\phi}\right) & =-\Lambda\left(\mathcal{E}^{\psi, \phi, 1}, 1\right)+i \sqrt{N_{2}} \tau(\psi) a_{\bar{\psi}} a_{\phi} \log \left(\frac{N_{1}}{N_{2}}\right) .
\end{aligned}
$$

where $\psi$ and $\phi$ are odd, primitive Dirichlet characters modulo $N_{1}$ and $N_{2}$ with $N_{1} \mid N$ and $\mathrm{N}_{2} \mid N$ and $\beta$ is extended linearly. Then

$$
\alpha=\beta \circ j .
$$




\section{Acknowledgements}

We are grateful to Kathrin Bringmann for drawing our attention to [7] and for many interesting discussions and to Don Zagier for many valuable comments on an early form of the note. We also thank Francois Brunault for reading carefully the submitted version of the paper and for offering very useful feedback. Finally we would like to thank the referee for very helpful comments that improved the exposition of the paper.

Received: 24 April 2015 Accepted: 27 October 2015

Published online: 21 December 2015

\section{References}

1. Daughton, A: A Hecke correspondence theorem for automorphic integrals with infinite log-polynomial sum period functions. Int. J. Number Theory. 10, 1857-1879 (2014)

2. Diamond, F, Shurman, J: A First Course in Modular Forms, Vol. 228. Springer-Verlag, New York (2005)

3. Goldfeld, D: Special values of derivatives of L-functions. Number theory (Halifax, NS, 1994), 159-173, CMS Conf. Proc., 15, Amer. Math. Soc., Providence, RI (1995)

4. Iwaniec, H: Topics in Classical Automorphic Forms. Graduate Studies in Mathematics, 17. American Mathematical Society, Providence, Rl (1997)

5. Kohnen, W, Martin, Y: Products of two Eisenstein series and spaces of cusp forms of prime level. J. Ramanujan Math. Soc. 23, 337-356 (2008)

6. Rubinstein, M, Sarnak, P: Chebyshev's bias. Experiment. Math. 3(3), 173-197 (1994)

7. Zudilin, W: Period(d)ness of L-values. Number theory and related fields, Springer Proc. Math. Stat, Vol. 43, pp. 381-395. Springer, New York (2013)

\section{Submit your manuscript to a SpringerOpen ${ }^{\odot}$ journal and benefit from:}

- Convenient online submission

- Rigorous peer review

- Immediate publication on acceptance

- Open access: articles freely available online

- High visibility within the field

- Retaining the copyright to your article

Submit your next manuscript at $\gg$ springeropen.com 